\title{
Post COVID-19 tuberculosis: An emerging threat of pandemic
}

\author{
Aqusa Zahid ${ }^{1}$, Nousheen Iqbal ${ }^{1,2}$, Sarosh Moeen ${ }^{3}$, Muhammad Irfan' \\ ${ }^{1}$ Department of Medicine, Section of Pulmonology and Critical Care, Aga Khan University Hospital, Karachi; ${ }^{2}$ Jinnah \\ Medical and Dental College, Karachi; ${ }^{3}$ Department of Pathology and Laboratory Medicine, Aga Khan University \\ Hospital, Karachi, Pakistan
}

\begin{abstract}
Severe acute respiratory syndrome coronavirus 2 (SARSCoV-2) disease (COVID-19) pandemic has hit the world hard. Millions of people have died due to the infection and several have suffered with what are now known as post COVID-19 squeal. Among these squeals one is immunosuppression which leaves patients prone to severe opportunistic infection. We here report a case of young female who was infected by COVID-19 and later developed cavitary pneumonia which upon investigation turned out to be due to mycobacterium tuberculosis. Through this report we aim to highlight the importance of high index of suspicion for infection like Mycobacterium tuberculosis after COVID-19 infection which developed in a healthy immunocompetent patient.
\end{abstract}

Correspondence: Nousheen Iqbal, Department of Medicine, Section of Pulmonology and Critical Care, Aga Khan University Hospital,

Karachi, Pakistan. E-mail: naush.akuh@gmail.com

Key words: Tuberculosis; post COVID-19; lung infections.

Contributions: All the authors made a substantive intellectual contribution, performed part of the experiments. All the authors have read and approved the final version of the manuscript and agreed to be accountable for all aspects of the work.

Conflict of interest: The authors declare that they have no competing interests, and all authors confirm accuracy.

Ethics approval: Not required.

Informed consent: obtained from the patient.

Received for publication: 24 December 2020.

Accepted for publication: 26 January 2021.

${ }^{\circ}$ Copyright: the Author(s), 2021

Licensee PAGEPress, Italy

Monaldi Archives for Chest Disease 2021; 91:1749

doi: 10.4081/monaldi.2021.1749

This article is distributed under the terms of the Creative Commons Attribution Noncommercial License (by-nc 4.0) which permits any noncommercial use, distribution, and reproduction in any medium, provided the original author(s) and source are credited.

\section{Introduction}

Mycobacterium tuberculosis affected 10 million people worldwide in the year 2019, Pakistan is among the eight high TB burden countries according to the world health organization (WHO) report. Lungs are the most commonly infected organ by mycobacterium tuberculosis. One of the most common risk factors of development of the disease is immunosuppression [1]. Ever since pandemic of COVID-19 hit the world a lot of opportunistic infection have surfaced most commonly reported of which are invasive aspergillosis and pneumocystis infection [2,3]. Although Mycobacterium tuberculosis has not been reported extensively as post COVID-19 infection, a significant number of reports have predicted rise in the incidence of disease due to current pandemic. A few notable studies include Tadolini et al. with a cohort of 49 cases, Nitesh et al. with a group of 22 patients and Motta et al. with 69 patients [4-7]. These series describe the characteristic of patient with COVI-19 and mycobacterium tuberculosis and their cause of mortality. However, none of these studies were able to establish a clinical mechanism behind the co-infection and establish a temporal relationship between the two. Moreover, most of the cases described above had either previously been treated for mycobacterium tuberculosis or had already been on treatment for it. Hence the aim of our report is to bring to attention the importance of high index of suspicion for the disease even in immunocompetent individuals without any risk factors for the disease like our patient.

\section{Case Report}

A 26-year-old female presented in pulmonology clinic with fever and hoarseness of voice for 1 month. The fever was high grade and associated with dry cough. She had a history of mild COVID- 19 pneumonia (Polymerase chain reaction PCR positive) before this illness and was managed at home in isolation with antibiotics and oral prednisolone $20 \mathrm{mg}$ twice daily for 14 days. The patient had improved clinically and remain asymptomatic. Before presentation in pulmonology clinic, she had consulted otolaryngologist who prescribed her azithromycin and voice rest but there was no improvement. On examination she was a young female with average height and build with hoarse voice. Chest auscultation revealed bronchial breath sound in right mid part of chest. Chest radiology was done and showed right mid zone cavitation (Figure 1). Her COVID -19 PCR was checked again and came out negative. As she was not producing sputum bronchoscopy was done. The bronchoscopy showed mobile inflamed 
vocal cords, the trachea was coated with white patches (Figure 2 a,b) till the right main bronchus, Bronchoalveolar lavage (BAL) was done from right upper and middle lobe and were sent for microbiological examination and Xpert MTB/Rif. Endobronchial biopsy was taken from the right middle lobe bronchial wall. The BAL Acid Fast Bacilli (AFB) smear and Xpert MTB/Rif came out to be positive without rifampicin resistance and the biopsy showed caseating granulomas (Figure 3). BAL Galactomanan came out 0.16 , AFB culture showed pan sensitive Mycobacterium tuberculosis while bacterial culture was negative. The patient was started on anti-tuberculous therapy and was followed up in the clinic. The patient was treated for tuberculosis disease with first line antituberculosis drugs including Isoniazid, rifampicin, pyrazinamide and ethambutol. The patient improved clinically became afebrile, regained her voice and responded to treatment well Just after 2 weeks of therapy. She is currently in intensive phase of therapy.

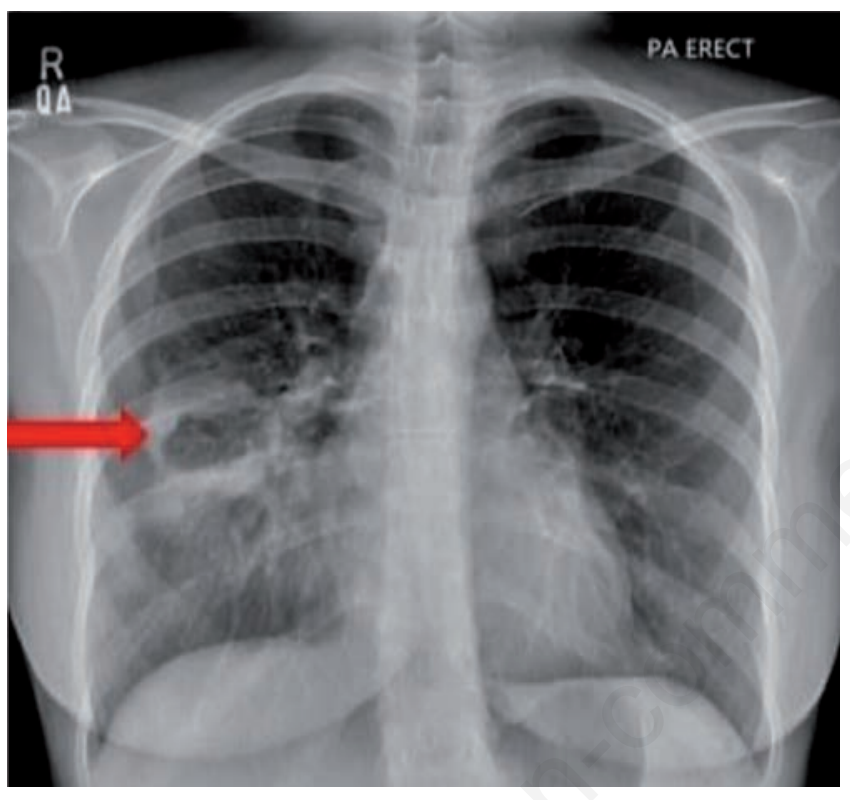

Figure 1. Chest radiograph showing cavity in right mid lung zone.

\section{Discussion}

COVID-19 pandemic has affected people extensively. Not only the virus itself has deleterious effects on the lungs but the immunosuppression caused by therapy for virus makes patients susceptible to a number of other opportunistic infections $[2,3]$. Tuberculosis is endemic in our part of world but the fact that COVID-19 infection increases its incidence is yet to be established. In first case series of a cohort of 49 patient published by Tadolini et al. [4] it was seen that 14 of these patients had a diagnosis of TB made after COVID-19 was diagnosed. However, they could not conclude whether COVID-19 lead to progression of latent $\mathrm{TB}$ to active $\mathrm{TB}$, other explanations presented by them were a possible co infection by both organism where COVID-19 was diagnosed first due to high index of suspicion or symptoms of COVID-19 brought into clinical evaluation of TB in otherwise asymptomatic TB infection. Our patient however had no evidence of symptomatic TB before COVID-19 infection, chest $\mathrm{x}$ ray had no cavitation and had acute onset of symptoms and that were suggestive of COVID infection. Development of active tuberculosis has been well reported in patients who have been immunocompromised either by viral infections like HIV or have comorbid conditions like diabetes or are on immunosuppressive agents like steroids that render their immune system week hence more prone to bacterial infection [8]. More studies that can identify a temporal relationship between the two infections are required. The COIVD-19 pandemic has been projected to bring forward detrimental consequences for TB. It has been estimated that current settings of lock down will limit diagnosis treatment and preventive measure of tuberculosis resulting in at least 6.3 million additional cases of tuberculosis between the year 20202025. It is speculated that at least 5 years of progress made towards TB elimination can be lost [9-11]. This impact was studied in an observational study done by Giovani et al at 37 TB centers located in 16 countries. It was found that during the early period of the pandemic a number of aspects of TB management have undergone significant setbacks. Some of the areas highlighted included decreased access due to transport interruption, fear of exposure to COVID-19, low priority given to TB screening [12]. The above facts are alarming and demand vigilance in our diagnosis of tuberculosis in patient who present with symptoms of tuberculosis.
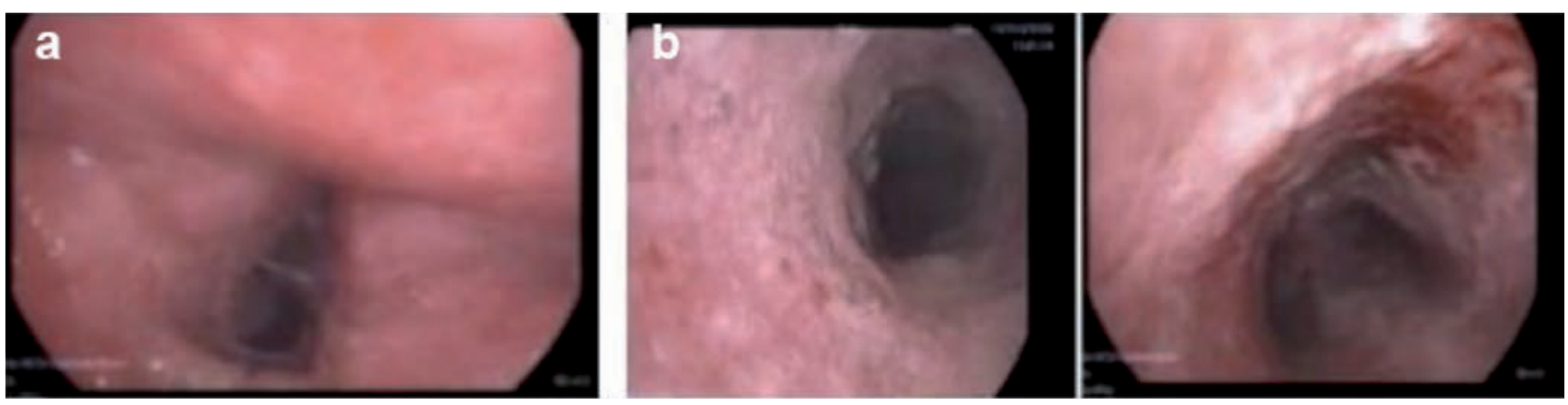

Figure 2. a) Inflamed vocal card; b) abnormal bronchial mucosa. 


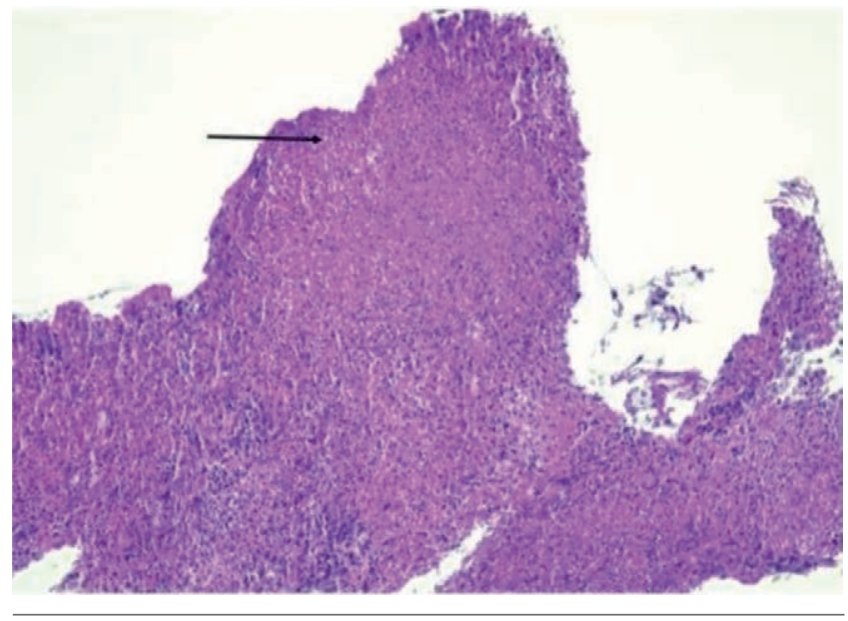

Figure 3. Histopathology of lung showing Granuloma formation with central caseating necrosis (arrow) surrounded by epithelioid histiocytes and lymphocytes. H\&E stain, original magnification x 100.

\section{Conclusions}

COVID-19 pandemic may lead to increase in TB cases due to reactivation of latent $\mathrm{TB}$ or new infection secondary to use of immunosuppressive medication or post viral immune function abnormalities. Tuberculosis should be in a differential diagnosis in post COVID-19 cases when someone presented with respiratory symptoms and radiological abnormalities.

\section{References}

1. Harding E. WHO global progress report on tuberculosis elimination. Lancet Respir Med. 2020;8:19.

2. Bartoletti M, Pascale R, Cricca M, et al. Epidemiology of invasive pulmonary aspergillosis among COVID-19 intubated patients: a prospective study. Clin Infect Dis 2020:ciaa1065.

3. Choy CY, Wong CS. It's not all about COVID-19: Pneumocystis pneumonia in the era of a respiratory outbreak. J Int AIDS Soc 2020;23:e25533.

4. Tadolini M, Codecasa LR, García-García JM, et al. Active tuberculosis, sequelae and COVID-19 co-infection: first cohort of 49 cases. Eur Respir J 2020;56:2001398.

5. Gupta N, Ish P, Gupta A, et al. A profile of a retrospective cohort of 22 patients with COVID-19 and active/treated tuberculosis. Eur Respir J 2020;56:2003408.

6. Rakesh PS, et al. Mortality among individuals with TBCOVID-19 coinfection: initial observations from Kerala, India. Int J Tuberc Lung Dis 2021; in press

7. Motta I, Centis R, D'Ambrosio L, et al. Tuberculosis, COVID19 and migrants: preliminary analysis of deaths occurring in 69 patients from two cohorts. Pulmonology 2020;26:233-40.

8. Narasimhan P, Wood J, MacIntyre CR, Mathai D. Risk factors for tuberculosis. Pulm Med 2013;2013:828939.

9. Zumla A, Marais BJ, McHugh TD, et al. COVID-19 and tuberculosis - threats and opportunities. Int J Tubercul Lung Dis 2020;24:757-60.

10. Alagna R, Besozzi G, Codecasa LR, et al. Celebrating world tuberculosis day at the time of COVID-19. Eur Respir J 2020;55:2000650.

11. Dara M, Sotgiu G, Reichler MR, et al. New diseases and old threats: lessons from tuberculosis for the COVID-19 response. Int J Tuberc Lung Dis 2020;24:544-5.

12. Migliori GB, Thong PM, Akkerman O, et al. Worldwide effects of coronavirus disease pandemic on tuberculosis services, January-April 2020. Emerg Infect Dis 2020;26:2709. 
